\title{
A Novel Chaotic-Speed Single-Phase Induction Motor Drive for Cooling Fans
}

\author{
Y. Gao, K.T. Chau and Shuang Ye \\ Department of Electrical \& Electronic Engineering \\ The University of Hong Kong \\ Hong Kong, China \\ ygao@eee.hku.hk, ktchau@eee.hku.hk, shuangye@eee.hku.hk
}

\begin{abstract}
This paper presents a novel chaotic-speed control of single-phase shaded-pole induction motor drives, especially for application to cooling fans. It is the firstly proposed and implemented chaotic-speed fan. Based on the $d-q$ axis model of the shaded-pole induction motor drive, Poincaré mapping and hence bifurcation analysis are conducted to reveal the periodic and chaotic operations under different system parameters. Consequently, a chaotic-speed fan can be achieved by properly choosing either the motor's own parameters or the operation condition such as the frequency and amplitude of the applied voltage. Theoretical analysis, computer simulation and experimental results are given to testify the proposed chaoticspeed fan.
\end{abstract}

Keywords—single-phase; shaded-pole; bifurcation; chaos

\section{INTRODUCTION}

Chaos is a phenomenon that occurs in many disciplines, from as large as the universe to as tiny as a particle. In electrical engineering, the nonlinear circuits, power systems, power converters, motor drives, telecommunications and medical electronics have already been identified to exhibit chaotic behaviors. Recent research has also been extended to the identification and hence the stabilization of chaos in different motor drives, especially DC and switched reluctance types [1], [2]. Rather than negatively avoiding the occurrence of chaos in motor drives [3]-[4], a positive idea is newly born the utilization of chaotic motion in motor drives.

It is well known that a variable-speed fan can provide a better cooling effect than its constant-speed counterpart. Recently, it has been identified that a proper chaotic motion can greatly enhance the efficiency in heat transfer [5]. Thus, it is anticipated that a chaotic-speed fan can further improve the cooling effect. Possible controllable behaviors are the chaotic boundaries and the types of chaos.

Since the shaded-pole induction motor (SPIM) is one of the most popular single-phase induction motors for cooling application [6], the purpose of this paper is to design and implement a novel chaotic-speed SPIM drive for application to cooling fans. Based on the $d-q$ axis model of the SPIM drive, Poincaré mapping and hence bifurcation analysis will be conducted to reveal periodic and chaotic operations under different system parameters. Consequently, the chaotic-speed fan can be achieved by choosing proper system parameters.

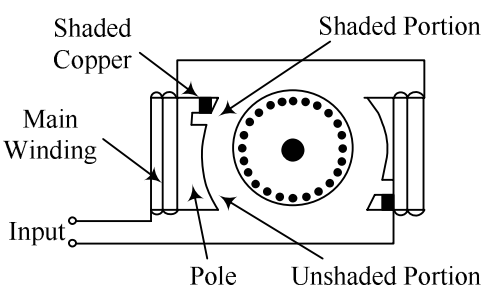

(a)

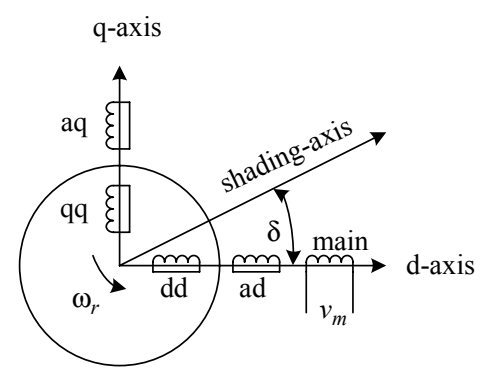

(b)

Figure 1. The SPIM: (a) Configuration; (b) $d-q$ axis model.

Theoretical analysis, computer simulation and experimental results will be given to testify the proposed chaotic-speed fan.

This paper is organized as follows: Section II will give a brief introduction to the SPIM, such as its advantages, applications, working principle and the mathematical model. The design principle and criterion for chaotic speed operation will be exploited in Section III. In Section IV, both numerical and experimental results using a practical SPIM will be presented to verify the proposed method. Section V will summarize the whole paper.

\section{SYSTEM MODELING}

The SPIM is widely accepted for domestic appliances, especially cooling fans. It offers the definite advantages of simple structure, low cost as well as highly rugged and reliable [6]-[7]. Its uniqueness is the use of the auxiliary winding, also called the shading winding, to produce a starting torque. As shown in Fig. 1 (a), a shaded-pole motor uses no starting switch. The stator poles are equipped with an additional

This work was supported and funded by a grant (under project no. HKU7154/04E) from Research Grants Council of Hong Kong Special Administrative Region, China. 
winding in each corner, that is, the shade winding. These windings have no electrical connection for starting but use induced current to make a rotating magnetic field. The shaded pole structure of the SPIM enables the development of a rotating magnetic field by delaying the buildup of magnetic flux. A copper conductor isolates the shaded portion of the pole forming a complete turn around it. In the shaded portion, magnetic flux increases but is delayed by the current induced in the copper shield. Magnetic flux in the unshaded portion increases with the winding current forming a rotating field. The interaction of these two magnetic fields generates the starting torque for the motor. Normally, the effect of the shading winding is negligible when the motor reaches speed [6].

Fig. 1 (b) shows the $d-q$ axis model of the SPIM. When the motor is connected to the power line, its main winding voltage has a form of $v_{m}=V \sin (2 \pi f t+\theta)$ where $V$ is the voltage amplitude, $f$ is the line frequency and $\theta$ is the initial phase difference with respect to $d$-axis. Then, the system dynamic behavior can be expressed as [8]:

$$
\begin{aligned}
& {\left[\begin{array}{c}
v_{m} \\
0 \\
0 \\
0
\end{array}\right]=\mathbf{L}\left[\begin{array}{c}
d i_{m s} / d t \\
d i_{a s} / d t \\
d i_{d r} / d t \\
d i_{q r} / d t
\end{array}\right]+\mathbf{R}\left[\begin{array}{c}
i_{m s} \\
i_{a s} \\
i_{d r} \\
i_{q r}
\end{array}\right]} \\
& \mathbf{L}=\left[\begin{array}{cccc}
L_{m m} & L_{m a} & L_{m d} & 0 \\
L_{m a} & L_{a a} & L_{a r} \cos \delta & -L_{a r} \sin \delta \\
L_{m d} & L_{a r} \cos \delta & L_{d d} & 0 \\
0 & -L_{a r} \sin \delta & 0 & L_{q q}
\end{array}\right] \\
& \mathbf{R}=\left[\begin{array}{cccc}
R_{m} & 0 & 0 & 0 \\
0 & R_{a} & 0 & 0 \\
0 & n_{p} \omega_{r} L_{a r} \sin \delta & R_{r} & -n_{p} \omega_{r} L_{q q} \\
n_{p} \omega_{r} L_{m d} & n_{p} \omega_{r} L_{a r} \cos \delta & n_{p} \omega_{r} L_{d d} & R_{r}
\end{array}\right] \\
& T_{e}=n_{p}\left[L_{m d} i_{m s} i_{q r}+L_{a r} i_{a s} i_{d r} \sin \delta\right. \\
& \left.+L_{a r} i_{a s} i_{q r} \cos \delta+\left(L_{d d}-L_{q q}\right) i_{d r} i_{q r}\right]^{\prime} \\
& J \frac{d \omega_{r}}{d t}=T_{e}-B_{m} \omega_{r}-T_{L}
\end{aligned}
$$

where $\mathbf{R}$ is the resistance matrix and $\mathbf{L}$ is inductance matrix; $i_{m s}$ and $i_{a s}$ are the currents of the main winding and shading winding, respectively; $i_{d r}$ and $i_{q r}$ are the rotor currents in the $d-$ axis and $q$-axis, respectively; $R_{m}, R_{a}$ and $R_{r}$ are the resistances of the main winding, the shading winding and the rotor, respectively; $L_{m m}, L_{a a}, L_{d d}$ and $L_{q q}$ are the self inductances; $L_{m d}$ is the mutual inductance between the main winding and $d$-axis rotor winding; $L_{m a}$ is the mutual inductance between the main winding and the shading winding; $L_{a r}$ is the mutual inductance between the shading winding and the rotor when the rotor is aligned with the shading winding; $\omega_{r}$ is the rotor angular speed; $n_{p}$ is the number of pole pairs; $\delta$ is the angle between the main winding and the shading winding; $J$ is the rotor inertia; $B_{m}$ is the viscosity friction coefficient; $T_{e}$ and $T_{L}$ are the electromagnetic torque and mechanical load, respectively.

\section{DESIGN FOR CHAOTIC-SPEED OPERATION}

The SPIM model described by (1)-(5) is a periodically driven non-autonomous system, which is apt to sub-harmonics resonance and chaos. The existence and stability of harmonic and sub-harmonic in such forced systems have been studied analytically and computationally in a number of biological and physiological applications. The most important examples are the periodically forced Van der Pol equation with damping [9], and oscillators of the Duffing type having a nonlinear restoring force [10]. Other usual used examples are the forced damped pendulums and springs.

In these driven harmonic oscillators, transience is found to lead to some steady state periodicity. The final behavior of the system depended on the relation between the driving frequency and the natural frequency (and to a lesser extent the damping factor). Similar behaviors are also found in first order nonlinear difference equations; the quadratic mapping and the related logistic equation. In the meanwhile, for such driven nonlinear oscillators, closed-form analytical solutions are usually not available and recourse must inevitably be made to numerical means.

In order to achieve the proposed chaotic-speed operation, a criterion for the existing of chaos becomes significant. Approaches by both motor parameter design and operation condition control have to be conducted based on this chaos criterion. Poincaré mapping and bifurcation analysis can be utilized for such a role.

For a system possessing more than one unique behavior, as a parameter is varied, an abrupt change in the steady-state behavior of the system is called a bifurcation. A plot of the steady-state obit against the bifurcation parameter is termed a bifurcation diagram. Thus, the bifurcation analysis facilitates the appraisal of the steady-state system behavior at a glance. In order to get such a bifurcation diagram, constructing a Poincaré map usually becomes quite necessary.

Poincaré mapping is an effective tool which functions to replace the solution of a continuous-time dynamic system by an iterative map. It acts like a stroboscope that produces a sequence of samples of the continuous-time solution. Thus, the steady-state behavior of the Poincare map, termed the orbit, corresponds to the steady-state waveform of the continuoustime dynamic system. For a non-autonomous system as described by (1)-(5), a natural way to construct the Poincaré map is to sample the trajectory with the applied line frequency $f$. Hence, the Poincaré surface $\sum \in \mathfrak{R}^{5} \times S^{1}$ can be defined as $\sum:=\left\{(\mathbf{x}, t) \in \mathfrak{R}^{5} \times S^{1}: t=t_{0}\right\}$ where $\mathbf{x}=\left[i_{m s}, i_{a s}, i_{d r}, i_{q r}, \omega_{r}\right]$ is the solution of the state vector. The trajectory of $\mathbf{x}(t)$ 
repeatedly passes the surface $\Sigma$ for every period $T$. The sequence of surface crossings, so-called the orbit, defines the Poincaré map as given by $\mathrm{P}: \mathfrak{R}^{5} \rightarrow \mathfrak{R}^{5}, \mathbf{x}_{n+1}=\mathrm{P}\left(\mathbf{x}_{n}\right)$ where $\mathbf{x}_{n}$ and $\mathbf{x}_{n+1}$ are the $n$th and $(n+1)$ th samples of $\mathbf{x}(t)$, respectively.

Bifurcation diagrams can be plotted once the orbits under different values of the bifurcation parameter are available. Since different types of attractor form different patterns on the Poincare surface, ignoring the case of the point attractor which has no intersection with the Poincare surface or just leave a single point without return, there are three main groups. Namely, a limit cycle forms one or several dots whose no corresponds to the period of the attractor. A quasi-periodic trajectory projects a ring or torus dot-cloud on the Poincaré plane, whereas chaotic attractors usually show relatively complex geometries with no apparent order. As a collection of these orbits, a bifurcation diagram reveals the change of the attractors with the variation of parameters. Based on the bifurcation diagram, one can easily design motor parameters or choose the right operation condition for chaotic or non-chaotic motion.

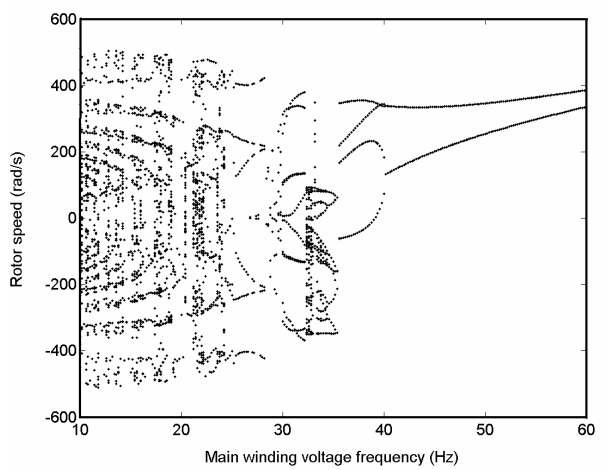

(a)

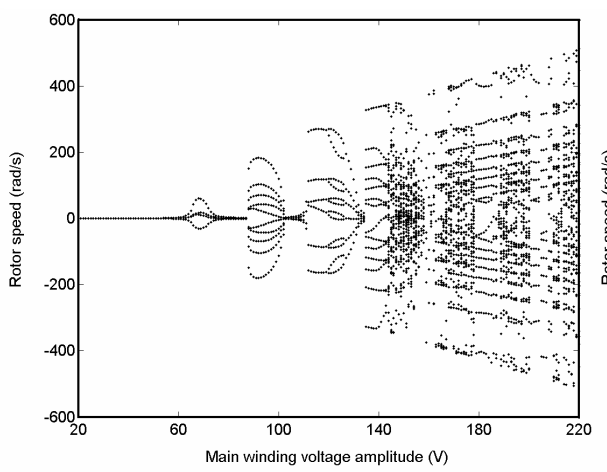

(b)

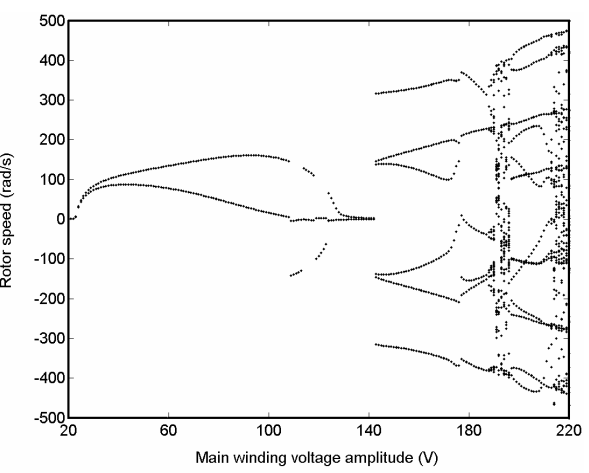

(c)

Figure 2. Speed bifurcation diagrams: (a) varying frequency with $V=220 \mathrm{~V}$; (b) varying amplitude with $f=10 \mathrm{~Hz}$; (c) varying amplitude with $f=22 \mathrm{~Hz}$.

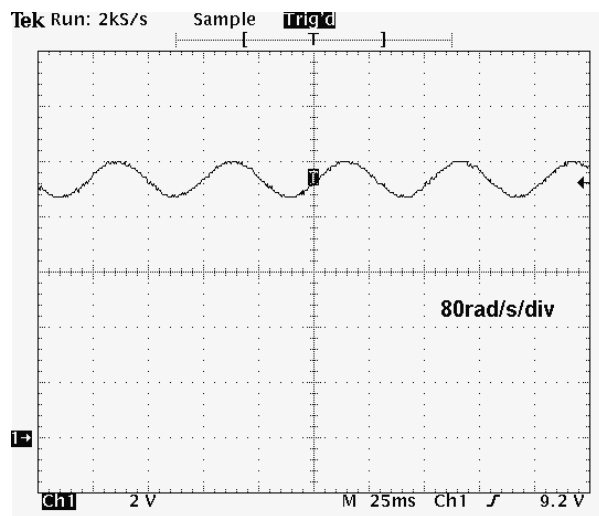

(a)

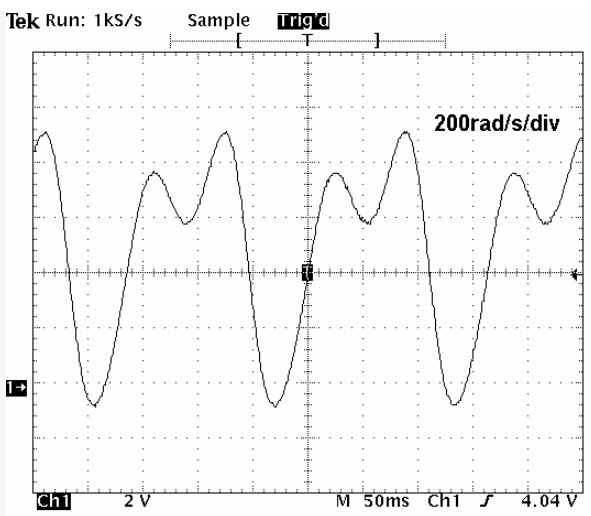

(b)

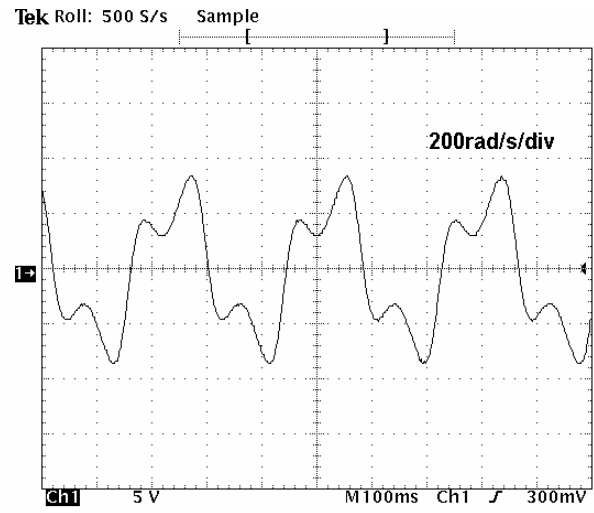

(c)

Figure 3. Measured periodic speed waveforms: (a) $V=220 \mathrm{~V}, f=60 \mathrm{~Hz}$; (b) $V=220 \mathrm{~V}, f=38 \mathrm{~Hz}$; (c) $V=160 \mathrm{~V}, f=22 \mathrm{~Hz}$.

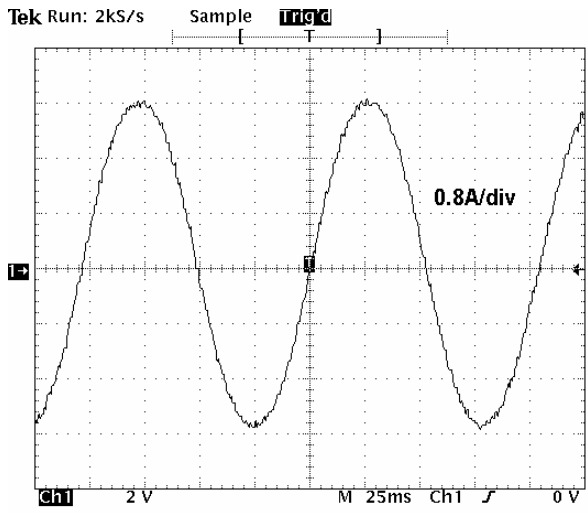

(a)

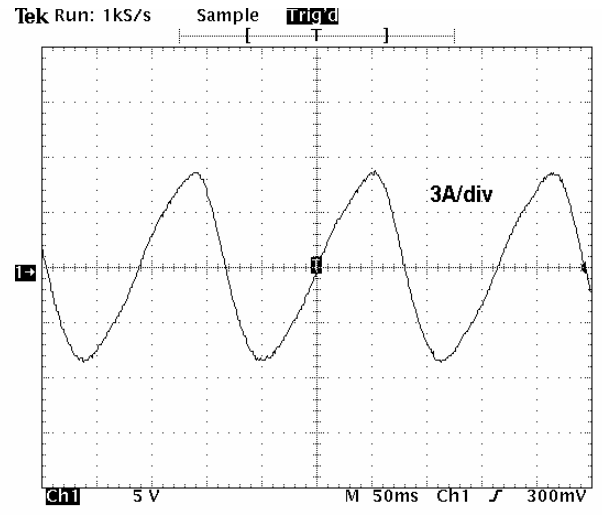

(b)

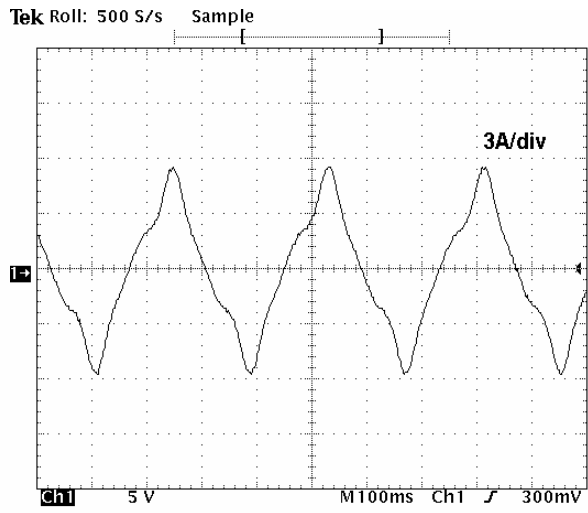

(c)

Figure 4. Measured periodic main winding current waveforms: (a) $V=220 \mathrm{~V}, f=60 \mathrm{~Hz}$; (b) $V=220 \mathrm{~V}, f=38 \mathrm{~Hz}$; (c) $V=160 \mathrm{~V}, f=22 \mathrm{~Hz}$. 


\section{EXPERIMENTAL RESULTS}

A practical SPIM drive with its motor parameters listed in Table $\mathrm{I}$ is used for exemplification. And $\theta=0$ and $T_{m}=0$ are used for all results. Based on the Poincaré mapping, speed bifurcation diagrams with respect to different system parameters can be obtained. Since the motor parameters, such as the pole numbers, inductances and resistances, are essentially fixed after production, the frequency and amplitude of the applied voltage are selected as the bifurcation parameters. The results are plotted in Fig. 2. Obviously enough, the motor behavior varies greatly with the change of operation parameters. Periodic oscillation, quasi-periodic and chaos can all be observed. Taking Fig. 2 (a) as an example, the speed bifurcation diagram shows that the motor acts like any other normal SPIMs in the frequency range of 40-60 Hz. Namely, it oscillates near a constant speed with a relative small oscillation amplitude, which is caused by the shading winding used for starting. With the decrease of the applied frequency, period-2 oscillation occurs. When the frequency drops further, complicated behaviors such as quasi-period and chaos arise. Despite of different patterns, similar conclusions can be cast for the rest of Fig 2. Therefore, different chaotic boundaries and even types of chaos can be resulted by varying either the frequency or the amplitude of the applied voltage.

Figs. 3 and 4 show the speed waveforms and the corresponding main winding current waveforms, respectively, at various periodic-speed operations, namely the period-1, period- 2 and period- 3 operations. It should be noted that the period-1 waveform shown in Fig. 3 (a) is the normal speed waveform of the conventional SPIM drive, whereas, the period- 2 and period- 3 waveforms correspond to its abnormal subharmonic operations. These waveforms consist with the well-known phenomenon of inevitable torque pulsation hence speed ripple in the SPIM caused by the shading winding.

TABLE I. MOTOR PARAMETERS

\begin{tabular}{|l|l|l|l|}
\hline \multicolumn{1}{|c|}{ Parameters } & \multicolumn{1}{c|}{ Value } & Parameters & \multicolumn{1}{c|}{ Value } \\
\hline$n_{p}$ & 1 & $R_{m}$ & $5.630 \Omega$ \\
\hline$L_{m m}$ & $0.4111 \mathrm{H}$ & $R_{a}$ & $0.212 \Omega$ \\
\hline$L_{d d}$ & $0.4105 \mathrm{H}$ & $R_{r}$ & $25.0 \Omega$ \\
\hline$L_{q q}$ & $0.3767 \mathrm{H}$ & $L_{a a}$ & $1.102 \times 10^{-5} \mathrm{H}$ \\
\hline$L_{m d}$ & $0.3528 \mathrm{H}$ & $\delta$ & $28^{\circ}$ \\
\hline$L_{m q}$ & $0.3448 \mathrm{H}$ & $J$ & $2.130 \times 10^{-5} \mathrm{~kg} \cdot \mathrm{m}^{2}$ \\
\hline$L_{m a}$ & $0.0015 \mathrm{H}$ & $B_{m}$ & $1.470 \times 10^{-4} \mathrm{~N} \cdot \mathrm{m} \cdot \mathrm{s}$ \\
\hline$L_{a r}$ & $0.0017 \mathrm{H}$ & & \\
\hline
\end{tabular}

As reflected from the speed bifurcation diagrams, the SPIM drive exhibits chaotic behavior at certain ranges of bifurcation parameters. Figs. 5 and 6 show the speed waveforms and the corresponding main winding current waveforms, respectively, at various chaotic-speed operations. It can be found that the chaotic speed waveforms offer the well-known chaotic properties, namely random-like but bounded oscillations. Also, these waveforms are aperiodic and very sensitive to the initial condition. Although, the main winding current waveforms seem to be more regular than the speed waveforms, they are still random-like and aperiodic when compared carefully. Physically, these chaotic motions reveal the unbalanced status of the interaction between the magnetic fields by the main winding and the shading winding, which is a kind of starting failures.

By this way, a chaotic-speed SPIM fan is realized. The key is to locate the motor behavior into the chaotic region of the bifurcation diagram by either motor parameter design or operation condition control. It should be noted that once the operation condition is known, the motor parameter whose variation bringing chaos may be more than one. Possible candidates are the main winding and the shading winding angle $\delta$, the mutual inductance $L_{a r}$, the ratio of $B_{m}$ to $J$, and so on However, to introduce chaos by choosing operation condition merely may fail for a given motor.

The proposed chaotic motion can be adopted to improve heat transfer and hence the cooling effect for homogeneity.

\section{CONCLUSION}

This paper firstly proposed and implemented a novel chaotic-speed SPIM drive for application to cooling fans. Based on the $d-q$ axis model of the SPIM drive, Poincaré mapping and hence bifurcation analysis has been conducted to reveal periodic and chaotic operations under different system parameters. Consequently, the chaotic-speed fan can be achieved by choosing proper system parameters such as motor parameters and operation conditions. Theoretical analysis, computer simulation and experimental results have been given to testify the proposed chaotic-speed fan. The tested results of a practical SPIM drive showed that the proposed method is fleasible enough for special applications desiring chaotic motions such as cooling fans or grinding machines.

\section{REFERENCES}

[1] J.H. Chen, K.T. Chau and C.C. Chan, "Analysis of chaos in currentmode controlled dc drive systems," IEEE Transactions on Industrial Electronics, vol. 47, pp. 67-76, 2000.

[2] K.T. Chau and J.H. Chen, "Modeling, analysis and experimentation of chaos in a switched reluctance drive system," IEEE Transactions on Circuits and Systems - I, vol. 50, pp. 712-716, 2003. 


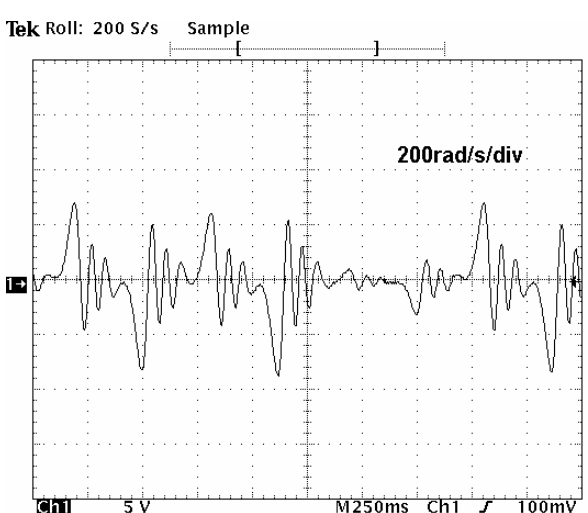

(a)

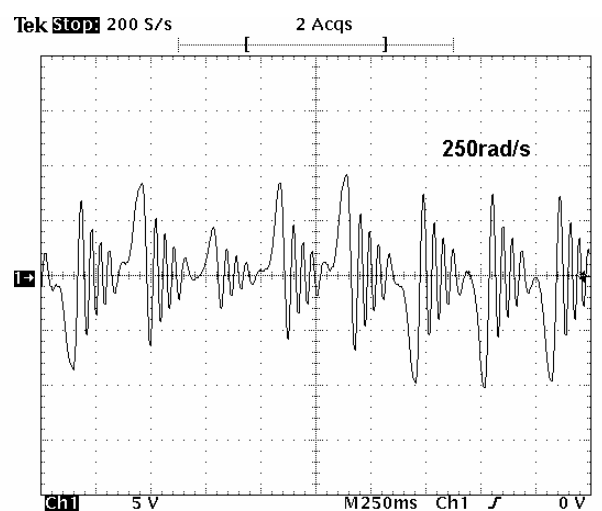

(b)

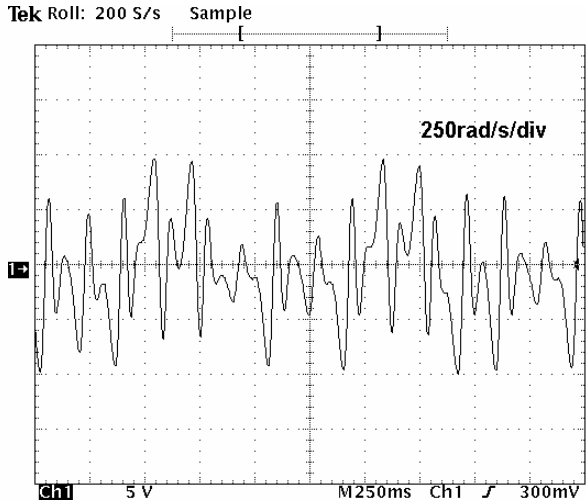

(c)

Figure 5. Measured chaotic speed waveforms: (a) $V=150 \mathrm{~V}, f=10 \mathrm{~Hz}$; (b) $V=220 \mathrm{~V}, f=10 \mathrm{~Hz}$; (c) $V=220 \mathrm{~V}, f=18 \mathrm{~Hz}$.

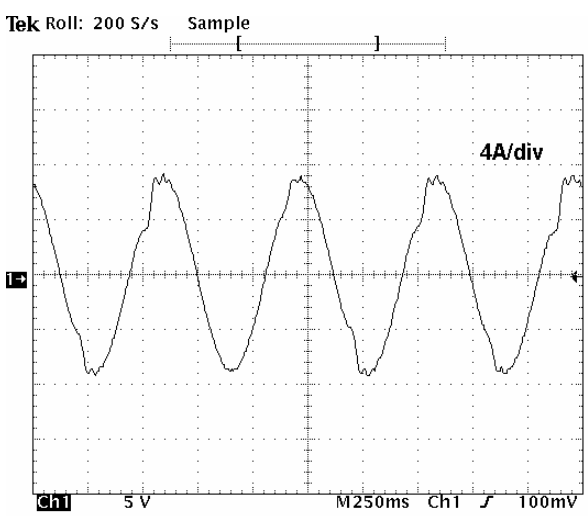

(a)

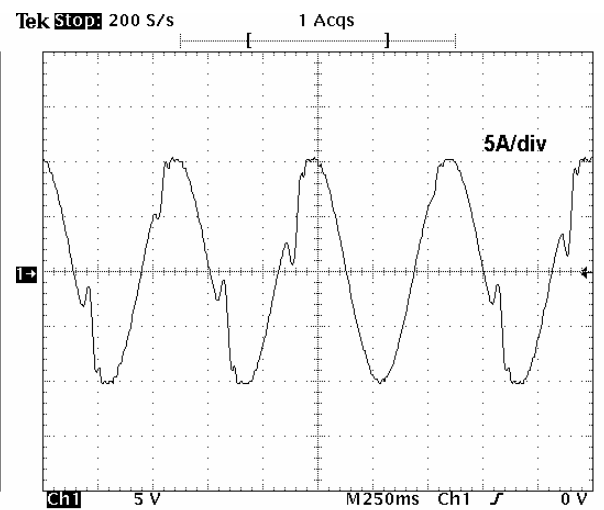

(b)

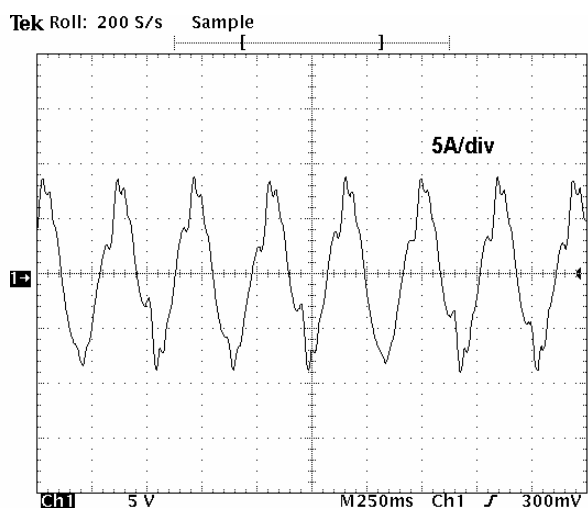

(c)

Figure 6. Measured chaotic main winding current waveforms: (a) $V=150 \mathrm{~V}, f=10 \mathrm{~Hz}$; (b) $V=220 \mathrm{~V}, f=10 \mathrm{~Hz}$; (c) $V=220 \mathrm{~V}, f=18 \mathrm{~Hz}$.

[3] Y. Gao and K.T. Chau, "Design of permanent magnets to avoid chaos in PM synchronous machines," IEEE Transactions on Magnetics, vol. 39, no. 5, pp. 2995-2998, 2003.

[4] Y. Gao and K.T. Chau, "Design of permanent magnets to avoid chaos in doubly salient PM machines," IEEE Transactions on Magnetics, vol. 40, no. 4, pp. 3048-3050, 2004.

[5] N. Acharya, M. Sen and H.C. Chang, "Heat transfer enhancement in coiled tubes by chaotic mixing," International Journal of Heat and Mass Transfer, vol. 35, pp. 2475-2489, 1992.

[6] C.G. Veinott and J.E. Martin, Fractional and Subfractional Horsepower Electric Motors. McGraw-hill, 1987.
[7] E. R. Collins, "Toque and slip behavior of single-phase induction motors driven from variable-frequency supplies," IEEE Transactions on Industry Applications, vol. 28, no. 3, pp. 710-715, 1992.

[8] A.M. Osheiba, K.A. Ahmed and M.A. Rahman, "Performance prediction of shaded pole induction motors," IEEE Transactions on Industry Applications, vol. 27, pp. 876-882, 1991.

[9] B. V. Pol and J. V. D. Merk, "Frequency demultiplication," Nature, vol. 120, pp. 363-364, 1927.

[10] F. C. Moon and P. J. Holmes, "A magnetoelastic strange attractor," Journal of Sound Vibration, vol. 65, pp. 285, 1979 